\section{NUCLEATION}

\section{Clusters and Yet More Clusters}

from our Molecular Physics Correspondent

Although some research fields seem to grow from a single, germinal experiment, others take root in accidents set off by workers stumbling from one preserve to another-and are none-the-less interesting for that. One might almost argue that a topic generated by coalescence of different interests is more ripe for progress than other more spectacular, though isolated, discoveries.

Nobody will pretend that the recent, intense activity centred on the detection and manipulation of small atomic and molecular clusters is likely to produce a very large salient at the frontiers of science-all the same it would be hard to find a neater and more stimulating set of experiments than those which have initiated this many-sided and completely original aspect of experimental rare-gas dynamics.

Three quite independent groups seem to share the honours in the crucial step, the discrimination and measurement of simple Van der Waals aggregates of 2, 3, 4 up to some 100 atoms. Priority goes to Becker, Bier and Henkes (Z. Physik., 146, 333 ; 1956) who noted the increase in intensity of nozzle beams due to cluster formation and performed the first mass spectrometric analysis of the products. At that stage, although the onset of nucleation in nozzle expansion was well known through the classic experiments of Oswatitsch in the 1940 s, nobody could be sure whether the measured cluster equilibria were established after the mass spectrometric ionization stage or were a truly thermodynamic effect. Two other laboratories contributed to the removal of these doubts, entering the subject with, it seems, a totally different viewpoint and objectives. Greene and Milne (J. Chem. Phys., 39, 3150; 1963) examined argon clusters from similar beams and were able to detect and mass-analyse nuclei as small as the dimer, while at about the same time Leckenby, Robbins and Trevalion (Proc. Roy. Soc., A, 280, 409; 1964) found clusters in the vapour of alkali metals, a system abounding in both practical and theoretical importance, from a conventional vapour effusion source.

The different points of departure seem worth recalling-Leckenby, Robbins and colleagues seem to have been involved with reactor engineering aspects of liquid sodium; Milne and Greene give their original interest as techniques of ion extraction from flames; Becker, Bier, Henkes and their collaborators give quite the most unexpected motive of the three--the eventual production of charged deuterium cluster ions for acceleration into thermonuclear machines. It seems a pity that little more has been heard of this project.

With the general techniques for obtaining observable concentrations of clusters now well established, more specialized attention has recently been devoted to the detailed explanation of the nozzle thermodynamics (Hagena and Obert, J. Chem. Phys., 56, 1793 ; 1972), to the kinetics of cluster growth and decomposition (Golomb, Good, Bailey, Busby and Dawbarn; J. Chem. Phys., 57, 3844 ; 1972) and to the measurement of special properties such as the ionization potentials of metallic systems (Foster, Leckenby and Robbins, J. Phys., B, 2, 478 ; 1969). Around all this a healthy body of theory has begun to grow, an idea of which might be given here by some representative ques- tions, all of them the idlest of speculation ten to fifteen years ago, but now well on the way to being experimentally decidable.

Is there a corresponding-states principle for different gases undergoing nozzle condensation or a related "corresponding jets" principle in the dynamics? (The answer is in the affirmative according to Hagena and Obert, with a possible reservation if quantum effects are involved.) Does the equilibrium concentration of dimers give more, or precisely as little, information about the interatomic potential as good second virial coefficient data? (More, insist Milne and Greene (J. Chem. Phys., $51,1685 ; 1969)$, replying to criticism with the point that the mass spectrometer samples stable clusters while the virial coefficients reflect averages over both these and metastable ones at higher energy.) How quickly does the ionization potential of, say, $\mathrm{Na}_{n}$ clusters fall to the work function of the bulk metal as $n$ increases? (It is about half-way

\title{
Ultraviolet Observations of Interstellar Mg
}

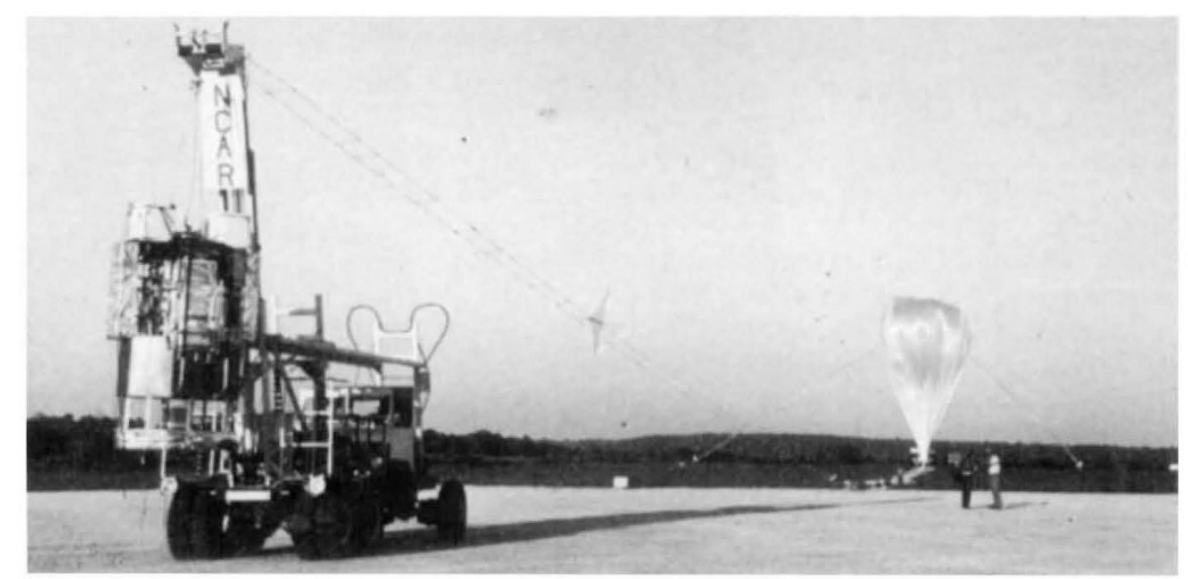

A BALLOON-BORNE objective grating spectrograph has been used to observe interstellar lines of $\mathrm{MgII}$ and $\mathrm{MgI}$ in the spectra of six stars. In next Monday's Nature Physical Science (December 11) Boksenberg and his colleagues describe the results of this joint experiment carried out under the auspices of University College, London, and Queen's University, Belfast, which has led to estimates of the interstellar electron density.

The balloon (see figure) was launched from Palestine, Texas, on October 4, 1972, and floated at an altitude between $42 \mathrm{~km}$ and $40 \mathrm{~km}$ for $12 \mathrm{~h}$; at these altitudes, zenith atmospheric transmission in the relevant part of the ultraviolet ( $\mathrm{Mg}$ lines are seen around $2800 \AA$ ) is about 50 per cent. Five of the stars observed were in Orion and the other in Cassiopeia; in general, the MgII lines were strong and the derived column densities of $\mathrm{Mg}^{+}$did not depend crucially on velocity models of the distribution in the line of sight. The cosmic abundance of $\mathrm{Mg}$ relative to $\mathrm{H}$ is $2.8 \times 10^{-5}$, and the values of magnesium abundance found for $\gamma \mathrm{Cas}$ and $\gamma$ Ori are close to that value. But for the three stars of Orion's belt, the abundances are rather higher.

The average value of the electron density for cool clouds in the line of sight to these five stars is $(1.5 \pm 0.6) \times$ $10^{-3} \mathrm{~cm}^{-3}$, assuming a cloud temperature of $60 \mathrm{~K}$, according to the calculations of Boksenberg et al.; because the derived values of this parameter are similar for all the observed stars they suggest that the best interpretation is that most interstellar absorption occurs in foreground cool clouds rather than in the vicinity of the stars. 